\title{
Technical review on derivation methods for behavior dependent functional responses
}

\author{
J. Garay
}

\author{
MTA Centre for Ecological Research, Evolutionary Systems Research Group, Klebelsberg Kunó u. 3, \\ H-8237 Tihany, Hungary \\ and \\ MTA-ELTE Research Group in Theoretical Biology and Evolutionary Ecology and Department of Plant Systematics, \\ Ecology and Theoretical Biology, ELTE Eötvös Loránd University, Pázmány Péter sétány1/c, H-1117 Budapest, \\ Hungary. Phone: +3613722500/8798,Fax:+3613812188.E-mail: garayj@caesar.elte.hu
}

Keywords: Markov chain, Renewal process, Stage dynamics, Time constraint, Wald's equality.

\begin{abstract}
Functional responses measure the trophic interactions between species, taking into account the density and behavior of the interacting species. In predator-prey interactions, the prey preference of the predator and the antipredator behavior of the prey together determine the feeding rate of the predator and the survival rate of the prey. Consequently, the behavior dependent functional responses make it possible to establish dynamic ecological models providing insight, among others, into the coexistence of predator and prey species and the efficiency of agents in biological pest control. In this paper the derivation methods of functional responses are reviewed. Basically, there are three classes of such methods: heuristic, stochastic and deterministic ones. All of them can take account of the behavior of the predator and prey. There are three main stochastic methods for the derivation of functional responses: renewal theory, Markov chain and the Wald equality-based method. All these methods assume that during the foraging process the prey densities do not change, which provides a mathematical basis for heuristic derivation. There are two deterministic methods using differential equations. The first one also assumes that during the foraging process the prey densities do not change, while the second one does not use that assumption. These derivation methods are appropriate to handle the behavior dependent functional responses, which is essential in the derivation of ecological games, when the payoff of prey and predator depends on the strategies of the prey and predator at the same time.
\end{abstract}

\section{Introduction}

Time constraint appears almost everywhere in biology, e.g., in different stages of ontogeny (such as offspring care, insect metamorphosis), territorial defense, fighting and sleeping, etc. In the review, I concentrate on the time constraint of feeding. The main point is that handling time can decrease the predation press on the prey population, since handling time decreases the number of active predators in the population. The aim of this technical review is to survey the different mathematical methods of the derivation of functional responses, mainly focusing on the effect of time constraints and the behavior of prey and predator. Consequently, this review does not intend to collect all functional responses that have been proposed (cf. Jeschke et al. 2002). Furthermore the question is not considered whether an already proposed functional response is viewed as reasonable from a biological viewpoint (see e.g., Fenlon and Faddy 2006, Jost and Ellner 2000). For instance, how to incorporate exactly the effect of predator density on the functional response remains somewhat controversial (e.g., Abrams 2014, Arditi et al. 1991, Arditi and Ginzburg 2012, Hossie and Murray 2016, Kalinoski and DeLong 2016, Kratina et al. 2009). Moreover, this review does not discuss the problem of conditions under which existing functional response can be applied to concrete biological cases (e.g., Tellez et al. 2009).

In the introduction, the following questions are considered: firstly, why is functional response one of the major issues in ecology? Secondly, why is the behavior of predator and prey crucial in the functional response, and what is the relationship between the functional response and optimization theory and/or game theory? Finally, a simple description of predation process is also given, which is the starting point of most derivation methods of functional responses.

\subsection{Why is behavior-dependent functional response important?}

Functional response (Solomon 1949) is the average number of food prey eaten by an arbitrary predator individual per unit time. In the broadest sense, the term "predator" means "forager", i.e., carnivore, omnivore, herbivore, parasites or parasitoids. In this paper, I will use the term predators in this sense. Similarly, in this general sense, prey will mean the food of the considered predator. Clearly, the functional response measures the negative effect of the predator species 
on the prey species. The numerical response (Solomon 1949) is the number of offspring (e.g., newborn, eggs or biomass growth rate) of a single predator per unit time. The numerical response depends on the prey eaten by a single predator. Thus it is a function of the functional response (cf. Garay et al. 2012, 2018). Consequently, functional response is the most essential component in trophic interactions, since it describes the intensity of the trophic connections.

Functional response is an important component in the following basic questions of theoretical ecology: when do trophically linked species coexist? Since all food webs are formed by trophically linked species, the question arises: when is a food web stable (Uchida et al. 2007, Valdovinos et al. 2010)? Now this question also arises: why are the behaviors of the prey and the predator important in this issue? In theoretical ecology, one of the possible mechanisms for maintaining diversity is negative frequency-dependent selection, i.e., rare prey experience higher survival than more common types (see e.g., Merilaita 2006, Punzalan et al. 2005). Clearly, in the predation process, predators' prey preference (cf. switching in the optimal foraging theory, Stephens and Krebs 1986, Krivan and Sikder 1999, and see Murdoch et al. 1975, van Baalen et al. 2001) implies negative frequency-dependent selection. Thus, the question arises: can the adaptive behavior of prey or predator stabilize the trophic interaction (Kondoh 2003, 2006, Valdovinos et al. 2010)? For an answer to the latter question, first we have to understand the effect of the adaptive behavior of prey or predator on functional response. For instance: What kind of prey preference maximizes the numerical response of a predator (see optimal foraging theory, Stephens and Krebs 1986)? Moreover, can the antipredator behavior of prey stabilize the food web (Kondoh 2007)? When is density dependent mutualism stable (Holland et al. 2002, Holland and DeAngelis 2010)? The above listed questions call the attention to the importance of the behavior dependent functional responses.

Moreover, the functional response is an important issue also from the view point of biological pest control. Does a given agent (predator, parasite species) effectively control the density of a given pest? (See e.g., Cabello et al. 2007, Lester and Harmsen 2002.) In biological pest control, the temperature dependence of the functional response is also important (Garcia-Martín et al. 2008), and the functional response of a given predator also depends on the prey types (Tellez et al. 2009).

In summary, the functional response is important in both theoretical and applied ecology.

\subsection{Aspects of a possible classification of functional responses}

Holling has already classified the functional responses according to the different linear and non-linear dependence on the density of a single prey species. The corresponding Holling types I-IV functional responses are well known (see Section 3.3).
This classification is based on the shape of the functional response. However, the predation process is not a simple one, thus the classification of the existing functional responses is not an easy task. For instance, Jeschke et al. (2002) classify and give the family tree of different 34 functional responses. Although the classification of all functional responses is not the aim of the present paper, I call attention to two aspects of functional responses. The main point is that functional response depends on the density and the behavior of prey and predator, simultaneously. Thus, from the point of view of the present paper, there are at least two basic aspects of a possible classification of functional responses:

Aspect 1. The density of how many species has effect on the given functional response (see e.g., Abrams and Ginzburg (2000): 1. The functional response is single prey dependent, when prey density alone determines the response (e.g., Holling I, II and III functional response, see Section 3.3). 2. The functional response is prey-predator dependent, when both predator and prey populations density affect the response (e.g., Beddington-DeAngelis functional response). 3 . The functional response is multispecies dependent, when species other than the focal predator and its prey species influence the functional response. In this paper we do not consider this case when a third species, namely parasite, modifies the functional response (Toscano 2014).

Aspect 2. Do the behaviors of interacting species have an effect on the given functional responses? The importance of Aspect 2 is that the theoretical models taking account of the behaviors of predators and prey have to use quite different mathematical tools to find the optimal behavior of predator and/or prey. Here there are four main possibilities.

2.1 No behavior effect. The behaviors of interacting species have no effect on functional response (see e.g., Holling II type in Section 2.1). This is the simplest case, there is no optimization problem and the functional response depends only on the density of prey.

2.2 Only predator's behavior has effect on its functional response without interference between predators. If there is no interaction between predators during the foraging process, and each predator has two different prey species, then the prey preference of the predator determines the functional responses (Stephens and Krebs 1986). The most studied problem in this case is how the predator's preference maximizes its numerical response. Optimal-foraging theory postulates that the forager maximizes its average net energy intake per unit time (Andersson 1981, Charnov 1976, Stephens and Krebs 1986, Turelli et al. 1982). The well-known zero-one rule of optimal foraging theory (either always attack or always ignore a prey type in the diet according to the actual density of different prey types) is one of the best examples for the way that predator behavior affects the functional response (Charnov 1976). A consequence of the zero-one rule is the negative frequencydependent selection, since the more abundant but less valuable prey type is attacked by the predator, if the density of the more valuable prey is low, so the predation pressure on the rare but more valuable prey type decreases. 
2.3. Only the prey's behavior has an effect on the predator's functional response. Clearly, the prey behavior can have an effect on the predator's success. Such kind of antipredator behavior is refuge using (Cressman and Garay 2009, Hossie and Murray 2010, McNair 1987, Sih 1987), crypsis (Erichsen et al. 1980), mimicry (Real 1977), herd formation (Cosner et al. 1999, Eshel 1978, Cressman and Garay 2011), habitat preference (Cressman et al. 2004) and active defense (e.g., Hammill et al. 2010). For instance, elephants and buffalos can actively defend themselves and their young against lions, and they can kill lions (Hayward and Kerley 2005).

It is important to point out here that there is an essential difference between the mathematical tools needed when the functional response exclusively depends on either prey or predator behavior.

When the predator's payoff does not depend on another predator's strategy and the preys' antipredator strategies, then the predator optimizes its foraging strategy (see classical optimal foraging theory, e.g., Stephens and Krebs 1986). When the prey use different antipredator strategies and the fixed predator's prey preference determines the survival rate of different antipredator strategy, then the survival rate of each prey type depends on the antipredator strategies used by the whole prey population (independently of whether predator can change its foraging strategy). In other words, the survival rate of a given prey type depends on the strategies used by other prey (Garay et al. 2015b); in which case a population game (Broom and Rychtar 2013, Brown and Vincent 1992) has to be used for the prey population (Garay et al. 2015b). I note that this selection situation for the prey is similar to apparent competition (Holt and Bonsall 2017) when the individuals from different prey species have no direct competition, but the common predator indirectly connects these species, since in one prey species the survival rate increases when the predator mainly consumes the other prey species. For an example of this kind of game, see Saleem et al. (2006) where, for a two predator one prey system, an evolutionarily stable strategy for the prey's defensive switching is given. Moreover, when Broom and Krivan (2018) surveyed the habitat selection game, they concentrated on functional responses of Holling types I and II.

2.4 Only predator's behavior has an effect on its functional response with interference between predators. If there is interaction between predators during the foraging process, then there are two cases. For the first case the BeddingtonDeAngelis functional response is a good example, where the interaction is not connected directly to the feeding, but the time constraint of the interaction decreases the total time duration of foraging, consequently the interaction time decreases the number of killed prey (see Section 3.3). For instance, the fight between predators clearly decreases the functional response (Garay at al. 2015a). In the second case, the interactions are connected to feeding. For instance, Auger et al. (2002) started from the classical hawk and dove game, when predator individuals can use two behavioral tactics to dispute a prey when they meet, and used Holling type I functional response. In kleptoparasitism (Iyengar 2008), fighting for the killed prey takes time, thus kleptoparasitism also decreases the predation pressure on prey. In summary, in the first case the optimization models are used to find the optimal behavior of the predator, while in the second case a game theoretical model is needed (Broom and Rychtar 2013).

2.5. The preys' and predators' behavior together have effect on the functional response. Clearly, Aspects 1 and 2 take place at the same time. For instance, the evolution of mimicry is a multi-species problem, where the predator's prey preference and the phenotypic similarity between two prey species together determine the evolutionary success of the different phenotypes of different prey species and the predator, as well (e.g., Getty 1985, Sherratt 2002). Furthermore, in Serengeti a pride of lions are hunting on gregarious prey and this group formation has an effect on the functional response (Fryxell et al. 2007, Krivan et al. 2008). If the preys' antipredator strategy and predators' prey preference have effect on the functional responses simultaneously, then a multi species, density dependent population game can be given by the behavior and density dependent functional response (Cressman et al. 2004, Garay et al. 2015b), since the prey and predator behavior together determine the functional response. Now I mention some game theoretical models for predator-prey interactions. Group formation has an effect on the survival of prey and energy gain of predator (Cressman and Garay 2011, Lett et al. 2004). Habitat usage also determines the predator's success (Cressman et al. 2004, Hammond et al. 2007, Holt 1985, Hugie and Dill 1994). Prey refuge usage increases the survival rate of prey (Molla et al. 2018, van Baalen and Sabelis 1993). Game theory is not only important in the predation process (e.g., Abrams et al. 1993, Brown et al. 1999, Vardi et al. 2017). For instance, in the plant-pollinator connection, the species are also counter-interested (e.g., Wang et al. 2012, Garay et al. 2003).

\subsection{Time constraint and predation cycle}

For the sake of simplicity (e.g., for the simplest figures and equations), here we consider only 3 main stages of the predation cycle, but these stages can be subdivided into further stages.

1.3.1. Search (traveling, encounter and recognition), when the predator is looking for its prey. This stage begins when the predator starts to look for food and ends when the predator finds its prey. Clearly, this stage includes the traveling, the local searching in a perception range and the recognition of prey type.

The traveling by predator is determined by the habitat preference of prey (Garay et al. 2018). There are two main factors determining the ideal free distribution of prey: firstly, the habitats give different food supply (Fretwell and Lucas 1970). Secondly, in different habitats the predation pressure is different according to the different properties of the habitat (Krivan and Schmitz 2003).

The local searching in a perception range also depends on the phenotype of the prey and the behavior of the predators. For instance, when the prey is cryptic, the predator can use its search image (Garay et al. 2018, Tinbergen 1960). 
The recognition of a prey type also depends on the phenotype of the predator and its prey (Kotler and Mitchell 1995). For instance, during the evolution of mimicry, the recognition of the palatable prey is an important factor (MacDougall and Dawkins 1998).

1.3.2. Attacks and killing. This stage starts when the predator attacks prey and ends when the predator has either killed or missed its prey. At this stage, the behaviors of the predator and its prey, together determine the success of the predator. Firstly, after the recognition of encountered prey, predator, according to its own prey preference, does or does not attack the prey. Secondly, the prey antipredator behavior has an effect on the success of the attack by the predator. For instance, refuge usage and gregarious behavior of the prey can also decrease the predator's success, and in some cases the active defense against predator is also successful.

1.3.3. Handling. This stage starts with either chop up, transport or eat and digest the food. During this stage the interaction between predators, e.g., kleptoparasitism (Wittenberger and Hunt 1985) or scrounge game (Barta and Giraldeau 2000) may happen.

Observe that a predation turn of the predator may end with several events: when the handling stage ends, when the predator has found not preferred prey, when the predator has missed the prey so the attacked prey survives, etc. The common feature of these three stages is time constraint, namely each predator spends time in these stages. After the predator has finished its turn, it starts a new turn, and so on, during the predation time duration $T$.

Based on the assumption that the different predator stages are mutually exclusive, the predation process form a predation cycle with different predation turns (see Fig. 1).

The structure of this paper is the following: Firstly, the heuristic derivation method is presented. Secondly, the stochastic methods are overviewed. Thirdly, the deterministic mathematical tools are surveyed. In each section a few applications are delineated, but a total overview is impossible, since there are too many of them. Finally, a short discussion is presented.

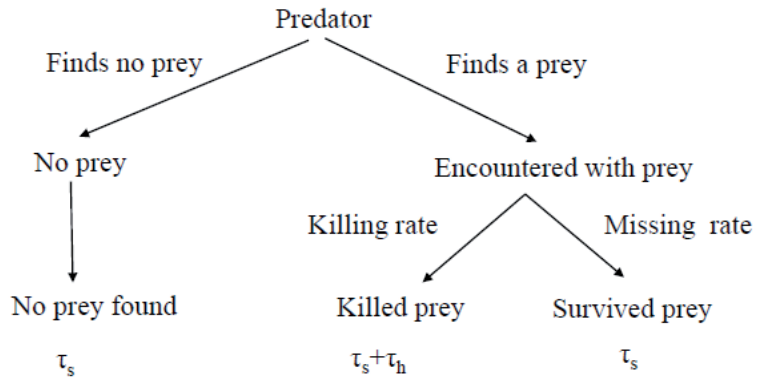

Figure 1. One turn of predation cycle, from starting a search to starting a next search, can be described by this tree. The searching and handling stages of the predator take time $\tau_{s}$ and $\tau_{h}$, respectively. More details of predation process are given in Lima and Dill (1990).

\section{Heuristic time-budgeting argument}

Since Holling used a heuristic argument when he introduced his functional response, I start with this derivation method. First, the original derivation of Holling is presented, then a more general heuristic reasoning is given.

\subsection{Holling's discs equation}

First we briefly recall the original derivation of Holling type II functional response by Holling (1959a,b). Consider a predator foraging during time duration $T$. The predator is either searching for a prey or handling a prey. Holling used the following assumptions:

A) The searching and handling exclude each other, and both need time: Let $\tau_{h}$ denote the average handling time of one prey individual. Then during $T$ we have two time durations: $T_{s}$ and $T_{h}$ the total time duration of searching and handling, respectively, so $T_{s}+T_{h}=T$.

B) Assume that during $T$ the finding rate of a prey does not change, let us denote it by $a$. We note that this assumption is valid if during $T$ the density of prey (denoted by $x$ ) is not changed by the predator.

C) There is a linear relationship between finding a prey and the prey density, i.e., if the prey density is doubled, the killing probability is also doubled. Formally, $p x$ is the killing rate if the prey density equals $x$ during $T$. We note that $p x$ is the average number of killings per time unit, and this is the reason why Holling's original derivation does not include the searching time.

Denoting the consumed prey by $y$, based on Assumption $\mathrm{C}$, we have

$y=p x T_{S}$

Indeed, since $p x$ is the average killing per unit time, during $T_{s}$ the number of killed prey is $p x T_{s}$. Moreover, since the handling time of a single prey, $\tau_{\mathrm{h}}$ determines the searching time, we also have

$T_{S}=T-\tau_{h} y$.

Substituting (2) into (1) we get

$y=p x\left(T-\tau_{h} y\right)$,

which simplifies to

$y=\frac{p x T}{1+p x \tau_{h}}$.

Thus, with $T=1$, we have

$y=\frac{p x}{1+p x \tau_{h}}$.

Although Holling (1959a) originally considered an artificial "predator-prey system": a human "predator" is searching for paper discs on the table, for the perspective of model building, in terms of the usual predation, Holling's model has the following important assumptions:

1. The predator's stages exclude each other, e.g., at a particular time each predator is either searching or handling but cannot do these two activities simultaneously. 
2. There is a time constraint, i.e., each disjoint action of predator needs time.

3. The killing rate depends on the density of prey.

4. During the investigated time period $T$, the density of prey does not change.

Although Holling's model is the simplest description of the problem, these motives of Holling's model are the basis of all mathematical derivation tools of functional response, reviewed below.

\subsection{Heuristic argument for fixed prey density}

There are three different time scales in the problem of predation. The first one is the longest one, the population growth time scale, which measures the densities change of predator and prey populations. This population dynamical time scale is significantly longer than that over which the functional response is measured (Abrams and Ginzburg 2000, Krivan and Cressman 2009). The second time scale, a smaller one is the time duration of predation cycles $T$. During $T$ the density change by predator can be neglected. The third time scale, the shortest one is the time unit for measuring the different time duration of different stages of the predation cycles. In most derivation methods of functional response, the population dynamics time scale does not take place.

Basic assumption: The following heuristic argument is strictly based on the widely used assumption that during $T$, the predator's behavior and the preys' density and the preys' behavior do not change (see later in Section 3). The definition of functional response (i.e., the average number of food prey eaten by an arbitrary predator individual per unit time), reads as

$F_{i}=\frac{\text { the average number of killed } i-\text { th prey during } T}{T}$.

What is the average number of killed $i$-th prey during $T$ ? For an answer to this question, let us consider what an observer can see? During $T$, each focal predator has a random sequence of predation turns and kills $i$-th prey in only part of these predation turns. Thus, the first question arises: what is the average number $G$ of different predation turns of a focal predator during $T$ ? For an answer to this question, let us calculate the average time duration of a predation turn (denote it by $E(\tau)$, which depends on the time duration of predation stages). In terms of $E(\tau)$, for $G$ we have

$$
G=\frac{T}{E(\tau)} \text {. }
$$

Now the second question arises: What is the probability $p_{i}$ that, in an arbitrary predation turn, the predator kills an $i$-th prey? Clearly, the average number of killed $i$-th prey during time $T$ equals $G p_{i}$, thus

$F_{i}=\frac{\text { the average number of killed } i-\text { th prey during } T}{T}=$

$=\frac{G p_{i}}{T}=\frac{p_{i}}{E(\tau)}$.

Hence we have
$F_{i}=\underline{\text { the probabilit } \mathrm{y} \text { of killed } i \text { - th prey one arbitrary predation turn }}$ the time average of one arbitray predation turn

Observe that this heuristic derivation is strictly based on the assumption that during $T$, all parameters of the predation process do not change, in other words, during $T$ these parameters are time independent. Indeed, in the opposite case, for instance when the prey density is radically decreased by predators during $T$, then the encounter probability also radically decreases, so firstly the average number of killed $i$-th prey is not equal to $G p_{i}$ (since the encounter probability decreases), and secondly the average time duration of one predation turn will increase (since the searching time increases).

Now, without a complete overview, I mention some papers where this derivation method is used. Spalinger and Hobbs (1992) started from the traditional representations of predator functional response and, by considering the plants distribution and the speed of herbivores, introduced new functional responses. The Beddington-DeAngelis functional response takes into account predator-predator interactions which also mutually exclude the searching and handling stage. The Beddington-DeAngelis functional response was derived by Beddington (1975), who extended the heuristic derivation method of Holling, and independently proposed by DeAngelis et al. (1975) based on an empirical relationship. Furthermore, Cosner et al. (1999), based on a timebudgeting argument and the principle of mass action, gave a unified mechanistic approach for the derivation of various forms of functional responses (including the ratio-dependent one), taking account of the spatial distribution of predators. Rogers (1972), using time-budgeting for parasites, derived a functional response in which the functional response is an exponentially saturating function of the host density. Pawar et al. (2012) pointed out that the dimensionality of consumer search space is probably a major driver of functional response, moreover, they also proposed functional responses for different foraging strategies (i.e., random active capture, sit-and-wait and grazing). Furthermore, Baker et al. (2010) and Smart et al. (2008) investigated the effect of vigilance on the functional response of granivorous prey. Anti-predator behaviors, including vigilance, are a major component of foraging (Treves 2000) and in some species it was shown that vigilance could become the major factor limiting feeding rate (e.g., Inger et al. 2006).

\section{Stochastic derivation methods}

In this section we overview the stochastic methods used in the derivation of functional responses.

\subsection{Renewal theory}

First, renewal theory was used to give proper mathematical foundations of functional responses (McNamara 1979, 1985, McNamara et al. 2006, Johns and Miller 1963, Oaten 1977, Oaten and Murdoch 1975, Pavlic and Passino 2011). Originally, the renewal process modeled the breakdown of 
different machines, and the renewal times represented the time between two consecutive breakdowns. In general, renewal theory focuses on random events occurring at random times, and the renewal time is the random time that elapses between two consecutive events. In essence, the application of renewal theory for the derivation of functional responses is based on Holling's assumption, namely, that during the predation time duration $T$, the density of prey does not change. Now a random event is that the predator kills a prey, and $I_{j}=1$ is the reward for it $(i=1, \ldots, n)$ the $i$-th prey, and the random renewal time is the random time duration between two kills (denoted by $\tau_{1}, \tau_{2}, \ldots, \tau_{n}$ ). $\tau_{i}$-s are supposed to be independent, identically (e.g., exponentially) distributed random variables, with finite mean, $E(\tau)<\infty$. Finally, the renewal process is that, after the kill the prey population "renewals". I note that, from the perspective of mathematics, this model is fine, but from biological view point it means that after one prey has been killed, exactly either one prey immigrates to the home range of predator or one prey is born. In other words, this application of renewal theory relies on the assumption that the processes of decrease and increase of the prey population during $T$ have the same effect on the local prey density.

Formally, the total time duration of $n$ killings is

$$
T_{n}=\sum_{i=1}^{n} \tau_{i}
$$

The renewal function is defined as the expected number of jumps, $m_{T}$, up to time $T$, i.e.,

$$
m_{T}=E\left(X_{T}\right),
$$

where $X_{T}$ is the number of events (rewards) up to time $T, X_{T}$ $=\sup \left\{n: T_{n} \leq T\right\}$.

The basic theorem of the renewal theory claims that

$$
\lim _{T \rightarrow \infty} \frac{m_{T}}{T}=\frac{1}{E(\tau)},
$$

which means that, if the total predation time duration $T$ is large enough, then the time average of a single killing determines the number of killings per unit time.

Moreover, the expected total reward during the predation time duration $T$, (in other words the reward function) is defined as

$$
F_{T}=E\left(\sum_{i=1}^{X_{T}} I_{i}\right),
$$

where $I_{i}$ denotes the indicator variable of the $i$-th event (in other words, obtained reward). The functional response (the number of killings during unit time) satisfies

$$
\lim _{T \rightarrow \infty} \frac{F_{T}}{T}=\frac{E(I)}{E(\tau)}
$$

Finally I note that the renewal theorem used here assumes that the time horizon is infinite.

Functional responses are often generated in optimal foraging theory using renewal theory (e.g., Andrews 1968, Cressman et al. 2014, Hassell 1978, Holling 1966, Houston and McNamara 1985, 1999, McNamara et al. 2006, Stephens and Krebs 1986, Yearsley 2003). Recently, Billiard et al. (2018) developed a bottom-up stochastic framework grounded in renewal theory showing how functional responses depend on the relative density of the individuals through the decomposition of interactions into different activities. They have derived the stochastic versions of classical functional responses: Holling's I, II and III. Furthermore, they demonstrated how the functional response depends on the dimension of foraging space, and body mass of prey. Finally, they also give the functional response when the prey have antipredator behavior, namely vigilance.

\subsection{Markov chains}

Markov chains have a wide range of applications in science, from physics to biology. Markov chain is "a stochastic model describing a sequence of possible events in which the probability of each event depends only on the state attained in the previous event" (Gagniuc 2017). In other words, Markov chain is "memoryless", i.e., the conditional probability distribution of future states of the process (conditional on both past and present states) depends only on the present state, not on the sequence of events that preceded it. This property of Markov chains is also called Markov property. Clearly, the basic assumption of Holling (see Section 2.1) that during the considered time period $T$, the density of prey does not change, gives a possibility for the application of Markov chains in predation processes as well.

A discrete-time Markov chain is a special sequence of random variables $X_{1}, X_{2}, X_{3}, \ldots$ The possible states of these random variables are the outcomes of a random phenomenon and run on a discrete set, so the possible values of $X_{i}$ form a finite set $S$ of $K$ elements (or more generally, a countable set). $S$ is called the state space of the chain. The above Markov property, namely that the probability of moving to the next state depends only on the present state and not on the previous states, for the discrete time case can be written as follows:

$$
\begin{aligned}
& P\left(X_{n+1}=x_{n+1} \mid X_{1}=x_{1}, X_{2}=x_{2}, \ldots, X_{n}=x_{n}\right)=P\left(X_{n+1}=\right. \\
& \left.=x_{n+1} \mid X_{n}=x_{n}\right), \text { if } \\
& P\left(X_{1}=x_{1}, X_{2}=x_{2}, \ldots, X_{n}=x_{n}\right)>0 .
\end{aligned}
$$

For the transition probability from i-th state to $\mathrm{j}$-th state introducing a shorter notation

$$
P_{i, j}=P\left(X_{n+1}=x_{j} \mid X_{n}=x_{i}\right),
$$

the so-called transition matrix $\mathbf{M}$ can be defined:

$$
\mathbf{M}=\left(\begin{array}{cccc}
P_{1,1} & P_{1,2} & \ldots & P_{1, K} \\
P_{2,1} & P_{2,2} & \ldots & P_{2, K} \\
\ldots & \ldots & \ldots & \ldots \\
P_{1, K} & P_{2, K} & \ldots & P_{K, K}
\end{array}\right) .
$$


Since the total transition probability from $i$-th state to all other states (including the $i$-th state) must be 1 , we have

$$
\sum_{j=1}^{K} P_{i, j}=1
$$

(In other words, $\mathbf{M}$ is a stochastic matrix). The main property of Markov chains is the existence of a stationary distribution $\mathbf{x}^{*}$, given by a probability row vector, which does not change under the application of the transition matrix M. In other words, it is a left eigenvector of matrix $\mathbf{M}$ associated with the eigenvalue 1, i.e.,

\section{$\mathbf{x} * \mathbf{M}=\mathbf{x}$.}

For the existence of a stationary distribution the notion of irreducibility is critical. We say that a Markov chain is irreducible if the probability of transition (even if in more than one step) from any state to any other state is not zero. Based on the theory of positive matrices (i.e., Perron-Frobenius theorem), it can be proved that every irreducible Markov chain has a unique stationary distribution.

Above we shortly summarized the basics of discrete Markov chains. However, the continuous time Markov chains are also widely used in applications. The main point is that, if all time durations are exponentially distributed, the state space is finite and the system is irreducible, then there always exists a unique stationary distribution (for the general proof see e.g., Garay et al. 2016). For the functional response, the predation state space is finite and the system is irreducible. Although the assumption that all time durations are exponentially distributed may be questionable, from the theoretical point of view this assumption can be considered as a simplifying one (e.g., Ruxton and Gurney 1994). I note that Kohlmann et al. (1999) fitted gamma distribution to the handling time for the herbivore (cf. Colombo 2013).

Van der Meer and Smallegange (2009), using a continuous time Markov model, derived a Beddington-DeAngelislike functional response taking into account agonistic interactions. Casas et al. (1993) used a Markov model for parasitoids. McCoy et al. (2011) introduced prey size dependent functional responses. Baudrot et al. (2016) for a generalist optimal forager investigated a multi-species functional response. Kannan (1983) used Markov chains for the predator's learning.

Finally I note the Markov chain is an essential mathematical tool in the time constrained evolutionary games (Broom et al. 2010, Garay at al. 2016) as well.

\subsection{Functional response based on Wald's equality}

Let us consider one predator and $k$ different prey types, and start from the assumption that during the predation time $T$, the densities of prey are constant, thus the probabilities of killing do not change during $T$. Let $\tau_{j}$ be the net predation time of $j$-th prey. Let the successive predation periods (times between consecutive kills) be $X_{1}, X_{2}, \ldots$; they are supposed to be independent, identically distributed, nonnegative random variables, with $P\left(X_{1}=\tau_{j}\right)=p_{j}, 1 \leq j \leq k$. That is, $p_{j}$ denotes the probability that the predator kills a prey of type $j$. Let us fix a positive $T$. We are interested in the (random) number $Z_{j}$ of predation periods, beginning before time $T$, when the prey is of type $j$. The following estimate for the expectation $E\left(Z_{j}\right)$ has been proved in Garay and Móri (2010)

$$
\frac{p_{j} T}{E\left(X_{1}\right)} \leq E\left(Z_{j}\right) \leq \frac{p_{j}\left(T+\tau_{m}\right)}{E\left(X_{1}\right)},
$$

where $\tau_{m}=\max \left\{\tau_{1}, \ldots, \tau_{k}\right\}$.

If $T$ is much greater than $\tau_{m}$, our formula (8) says that the average number of type $j$ killed per unit time is approximately equal to,

$$
\frac{p_{j}}{E\left(X_{1}\right)}
$$

which is heuristically quite clear: During a long time $T$ the number of predation periods is approximately equal to $T$ divided by the mean time of one such period; and the number of periods where type $j$ prey is killed is proportional to the probability $p_{j}$. Such a heuristic reasoning can be very useful for getting an insight, but leaves uncertainty about the goodness of the approximation. The significance of estimate (8) is that it attributes exact mathematical meaning to heuristic considerations. The proof of the above results is based on Wald (1944) equality (see Garay and Móri 2010).

For harmony with the already proposed functional responses, we assume that $\tau_{m} / T$ is small enough. Then formula (8) becomes

$$
E\left(Z_{j}\right) \cong \frac{p_{j} T}{E\left(X_{1}\right)},
$$

so the general formula for functional response is obtained (for the mathematical details see Garay and Móri 2010)

$$
F_{j}=\frac{p_{j}}{E(\tau)}
$$

which is the same as formula (7). The main point is that using Wald's equality we have a mathematical proof for the formula (7) under the assumption that, during $T$ the parameters of the predation cycle do not change.

I note that the main difference between renewal theory and Wald's equality based methods is that the first one considers infinite time horizon, while the second one considers finite time horizon. Furthermore, the second one gives an approximation of the functional response, see equation (8). Moreover, using Wald's method, Garay and Móri (2010) pointed out that prey preference is optimal whenever the advantage of a proper prey preference is larger than the average cost of missed prey preference.

To demonstrate the usefulness of our formula (10), we consider the following simplest predation cycle, where there is no behavior effect, thus the functional response depends only on the density of prey and the predator can kill only one prey at a given time (see Fig. 2). 


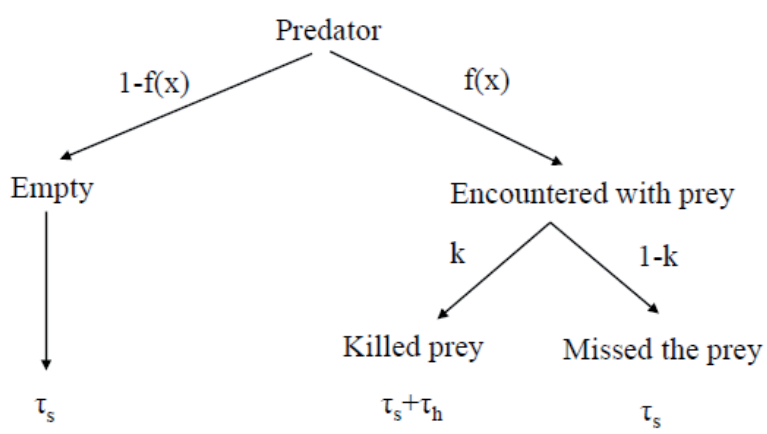

Figure 2. The density dependent encounter probability with prey is denoted by $f(x)$. The probability of a successful attack is denoted by $k$. If the encounter and attack are independent then the predator has killed its prey with probability $p=f(x) k$. The searching and handling stages of the predator take times $\tau_{s}$ and $\tau_{h}$, respectively.

Let $p$ be the probability that the predator kills its prey, and $\tau_{s}$ and $\tau_{h}$ be the searching and the handling time, respectively. For the application of formula (10), we have to give probability $p$. Clearly, $p$ depends on the accessibility of prey (which depends on the density of prey, the territorial structure of the predators, etc.) and the success of predation attacks at least. In the following, five applications are presented.

Application 1. Holling type II. Predator can observe one prey individual at most and prey are independently and uniformly distributed in perception ranges. Denote by $H$ the number of perception ranges, then a uniformly random searcher predator encounters its prey with probability

$$
f(x)=\frac{x}{H},
$$

where $x$ is the number of prey. Clearly $x<H$ is needed. Denote the probability of successful attacks by $k$. Then, we can assume that

$p=\frac{x}{H} k$

Now, formula (10) reads as Holling type II functional response:

$$
F=\frac{x \frac{k}{H}}{\tau_{s}+\tau_{h} x \frac{k}{H}}=\frac{x k}{\tau_{s} H+\tau_{h} x k} .
$$

Observe that the denominator here gives the average time duration of one predation turn, i.e. now

$$
E(\tau)=\tau_{s}+\tau_{h} x \frac{k}{H}
$$

Application 2. Holling type III. Now we will consider a theoretical case when predator perception is "preys' density" dependent: predator can observe a prey if there are two individuals at the same time in its search patch. If prey are independently and uniformly distributed in perception ranges and each predator can find a perception range with two prey, then the encounter probability is

$$
f(x)=\left(\frac{x}{H}\right)^{2} \text {. }
$$

Therefore now

$$
p=\left(\frac{x}{H}\right)^{2} k \text {. }
$$

Hence, formula (10) results in the Holling type III function response:

$$
F=\frac{\left(\frac{x}{H}\right)^{2} k}{\tau_{s}+\tau_{h}\left(\frac{x}{H}\right)^{2} k} .
$$

Application 3. Holling type $I V$. Now, assume that prey is not randomly distributed in the perception ranges, namely in a perception range there are 0,1 and 2 prey with probabilities

$$
1-\frac{x}{H}-\left(\frac{x}{H}\right)^{2}, \quad \frac{x}{H} \text { and }\left(\frac{x}{H}\right)^{2},
$$

respectively. Moreover, assume that the predator can kill its prey with probability 1 , if there is only one prey in perception range. In other words, two prey either can observe the stalker predator in time to flee, or two prey can successfully defend against predator. Furthermore, when the predator has found two prey in perception range then it needs an extra time denoted by $\tau_{2}$, and it cannot kill any prey. Under this condition the probability of killing is

$$
p=\frac{x}{H},
$$

and the average time duration is

$$
\begin{aligned}
& E(\tau)=\left(1-\frac{x}{H}\left(1-\frac{x}{H}\right)\right) \tau_{s}+\frac{x}{H}\left(\tau_{s}+\tau_{h}\right)+\left(\frac{x}{H}\right)^{2}\left(\tau_{s}+\tau_{2}\right)= \\
& =\tau_{s}+\frac{x}{H} \tau_{h}+\left(\frac{x}{H}\right)^{2} \tau_{2} .
\end{aligned}
$$

Now, formula (10) reads as the Holling type IV functional response (Andrews 1968, Boon and Landelout 1962, Edwards 1970). Indeed

$$
F=\frac{\frac{x}{H}}{\tau_{s}+\frac{x}{H} \tau_{h}+\left(\frac{x}{H}\right)^{2} \tau_{2}} .
$$

Application 4. Ratio dependent functional response. The ratio dependent functional response was introduced by Arditi and Ginzburg (1989), and there are evidences that the functional response in the wolf-moose system is ratio dependent (Jost et al. 2005, Vuchetich et al. 2002). Now, let us consider $y$ 
territorial predators and its prey living in a huge herd moving across the territory of all predators, and spending the same time duration in each territory. Under these conditions, we can assume that if the encounter probability depends on the size of the herd, i.e.,

$$
f(x)=\frac{x}{H y},
$$

then the probability of killing is

$$
p=\frac{x}{H y} k \text {. }
$$

Now, formula (10) provides the ratio dependent function response:

$$
F=\frac{\frac{x}{H y} k}{\tau_{s}+\frac{x}{H y} k \tau_{h}}=\frac{\frac{x}{y} k}{\tau_{s} H+\frac{x}{y} k \tau_{h}} .
$$

Application 5. Fighting predator. Let us suppose now that, if two predators encounter each other, then they fight. In this encounter, the fight does not only take time, but we can also assume that during fighting the occasional injury may happen, and during recovery the predators stop all other activities, including hunting (Garay et al. 2015a). Consider a prey and a predator species, and the following activity distribution: No interaction happens with probability

$$
p_{1}=1-\frac{x}{H}-\frac{y}{H}
$$

two predators fight with probability

$$
p_{2}=\frac{y}{H}
$$

a predator misses a prey with the probability

$$
p_{3}=\frac{x}{H}(1-k)
$$

and kills it with probability

$$
p_{4}=\frac{x}{H} k
$$

(See Fig. 3. I emphasize that these probabilities do depend on the density of the prey and the predators, since their encounter probabilities depend on the densities.) Denote by $\tau_{i}$ the average time duration of the above activities and let $\tau_{\mathrm{s}}$ be the searching time, namely, $\tau_{1}=\tau_{s}, \tau_{2}=\tau_{s}+\tau_{f}$, where $\tau_{f}$ denotes the average fighting time, $\tau_{3}=\tau_{s}$, for the sake of simplicity, assume the attacks do not take time, $\tau_{4}=\tau_{s}+\tau_{h}$, where $\tau_{h}$ denotes handling time. Now, formula (10) reads

$$
F=\frac{p_{4}}{\sum_{i=1}^{4} \tau_{i} p_{i}}=\frac{x k}{\tau_{s} H+\tau_{f} y++\tau_{h} x k} .
$$

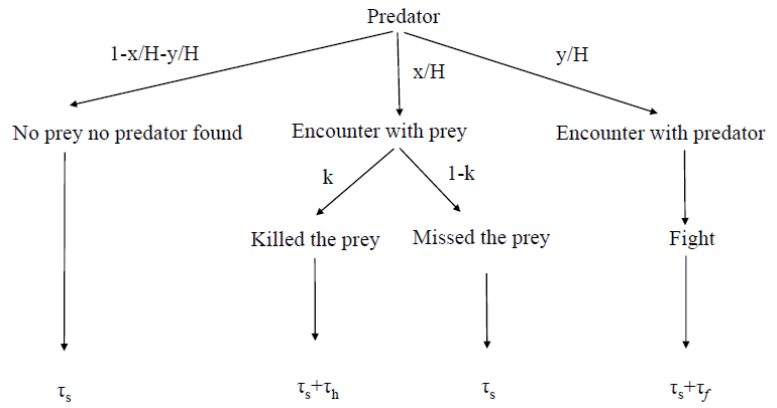

Figure 3. The interference between predators (in this example fight) decreases the time duration of foraging, so also decreases the functional response. Average activity time durations $\tau_{i}$ are described in the main text.

I note that the Beddington-De Angelis functional response for fighting predator was obtained by using the Wald's equality based method (Garay and Móri 2010). Furthermore, using Wald's equality based method for omnivore (Garay et al. 2012), Holling type II and III functional responses were obtained.

At first glance, the reader may think that this method is not quite new, since renewal theory (Johns and Miller 1963) and the heuristic time-budgeting argument give the same result: functional response is the amount of food items consumed on average, divided by the average duration time (see e.g., Houston and McNamara 1999, and the references therein). However, there are two main differences: the first one is rather technical; we used Wald's equality, which is a "vertical" description in time (considering a given time period in which the density change of prey can be neglected); unlike renewal theory, which is a "horizontal" description in time (assuming that time duration tends to infinity, and the prey density does not change, since it renews). Other authors consider renewal cycles with short renewal time period (e.g., McNamara 1985, McNamara and Houston 1999). However, if the prey population is quickly renewed (e.g., renewal time is shorter than searching time), then the predator will optimize energy intake by staying in the richest patch type once one is encountered and so obtain an expected payoff greater than random search by also decreasing the total searching time during $T$. I also note that if the predator never visits the same patch twice, then the basic condition of Wald's methods holds: if the prey is randomly distributed in the home range of predator, then the proportions of patch types among visited and non-visited patches are the same and are also unchanged during $T$. The second difference is more important in the derivation method based on Wald: although the $i$-th activity probability $\left(p_{i}\right)$ is fixed, it is not specified during the derivation. This gives a freedom for us to take account of the biological details of the considered situation, as we will see in the next section.

\subsection{Decision tree method}

In this subsection, we continue to use the assumption that during the predation time $T$, the densities of prey are constant, thus the probabilities of killing do not change during $T$. Now, we slightly generalize the model of Section 3.2. Let us consider $k$ different predation turns of a predation cycle. A 
predation turn starts with searching for prey, then continues with killing prey and ends with handling prey, etc., see Figure 1 . Let the net time of $j$-th predation turn be denoted by $T_{j}$. Let the times between consecutive predation turns be $X_{1}, X_{2}, \ldots$; they are supposed to be independent, identically distributed, nonnegative random variables, with $P\left(X_{1}=T_{j}\right)=p_{j}, 1 \leq j \leq k$. In this slightly generalized model, formulas (8) and (10) are also valid.

The decision tree method (Cressman et al. 2014) gives a simpler step-by-step "recipe" how we can take account of the details of the predation process like predator and prey behaviors, during the derivation of functional responses. There are three main successive steps of the predation cycle: the first stage is encounter, after which the predator does or does not attack its prey found, then killing and finally handling. Observe that these steps form a conditional sequence, since an attack can only occur if the predator has encountered its prey and so on. Furthermore, at each step there is a probability distribution, i.e., the encounters form a random process, moreover, if predator does not follow the 0 -1 rule, it randomly attacks its actually found prey, finally, in general predator can also miss its attacked prey with a given probability.

Decision trees for functional responses describe predator actions and prey behaviors at each decision point. When applied to functional responses, these trees must have three levels.

The first level describes the distribution of encounter events between predator and prey (cf. Fig. 4). In general, let us denote the different encounter events by $E_{i}$ and the probability that $E_{i}$ occurs by $P\left(E_{i}\right)$. The main point is that encounter event $E_{i}$ runs all situations where the predator has to make a decision. Since we also include events when the predator does not encounter any prey,

$$
\sum_{i} P\left(E_{i}\right)=1
$$

The encounter depends on the density and behavior of prey (Cressman and Garay 2011, Garay et al. 2015b), furthermore on whether the predator does or doesn't use search image (Dawkins 1971, Garay et al. 2015b). We note that during search, predator may have encountered another predator as well.

The second level describes the predator preference, let $\alpha_{k}$ give the predator's possible actions (i.e., what the predator does) in encounter $E_{i}$ and $s\left(\alpha_{k} \mid E_{i}\right)$ be the predator's conditional strategy of using action $\alpha_{k}$ when in encounter $E_{i}$ (see Fig. 4). We stress here that actions $\alpha_{k}$ are specific for each encounter event $E_{i}$ and so are more formally denoted by $\alpha_{k i}$. To simplify notation, the second index is omitted throughout this subsection. Then, for each $i, P\left[s\left(\alpha_{k} \mid E_{i}\right)\right] \geq 0$ and $\Sigma_{k} P\left[s\left(\alpha_{k} \mid E_{i}\right)\right]$ $=1$ where $P\left[s\left(\alpha_{k} \mid E_{i}\right)\right]$ is the probability of using conditional strategy $s\left(\alpha_{k} \mid E_{i}\right)$ in event $E_{i}$.

The third level describes the predator's success at killing its prey and may also depend on the encounter event $E_{i}$ and on its action $\alpha_{k}$ (see Fig. 4). Let $\kappa\left(\alpha_{k} \mid E_{i}\right)$ denote the probability that the predator is successful, which is 0 if $\alpha_{k}$ is an action that does not attack a prey and is a number between 0 and 1 otherwise.

For the general modeling approach, the "activity distribution" of the predator is introduced. An activity event $A_{\ell}$ is given by an encounter event $E_{i}$, an event specific action $\alpha_{k}$ and whether or not the predator kills its target (i.e., $A_{\ell}=\left\{E_{i}, \alpha_{k}\right.$, $\left.\left.\kappa\left(\alpha_{k} \mid E_{i}\right)\right\}\right)$. Taken together, all such events form a partition of the total event set. That is, every activity event is included in the union of the $A_{\ell}$ and two different $A_{\ell}$ and $A_{\ell}^{\prime}$ are mutually exclusive. The activity distribution answers the three questions posed at each stage of the predation process: encounter, predator's decision, predator's success. The probability $P_{\ell}$ of activity event $A_{\ell}$ is given through the information above. For example, if $A_{\ell}$ is the encounter event $E_{1}$ combined with action $\alpha_{1}$ and the predator kills the prey, then $P_{\ell}=P\left(E_{1}\right) P\left[s\left(\alpha_{1} \mid\right.\right.$ $\left.\left.E_{1}\right)\right] \kappa\left(\alpha_{1} \mid E_{1}\right)$. Since the functional response is based on the number of prey killed by the predator per unit time, we must also consider the duration $\tau_{\ell}$ of each activity event $A_{\ell}$.

Observe that the decision tree, by taking account of the sequences of conditional events, generates the distribution of activity (in other words predation turns) of a predator (see e.g., Garay et al. 2015b), and each activity has time duration (see Fig. 4). Suppose that the activity distribution is constant over a given time interval $T$. Then by applying formula (10) the average number of $A_{\ell 0}$ activity events per unit time, $F_{\ell 0}$, is given by

$$
F_{\ell}=P_{\ell} /\left(\sum \tau_{\ell} \mathrm{P}_{\ell}\right)
$$

Application 6. Two types of prey. The decision tree based game-theoretical approach (Cressman et al. 2014) was developed for constructing functional responses in multi-prey environments and for finding the corresponding optimal foraging strategies. Let us consider two different prey types $\mathrm{X}$ and $\mathrm{Y}$ with densities $x$ and $y$, respectively, and suppose that the different prey types do not aggregate, thus predator finds either X or Y prey, but never find an XY pair of prey. Assume that the encounter probability with $\mathrm{X}$ and $\mathrm{Y}$ prey is $x / H$ and $y / H$, respectively. If the predator has found prey $\mathrm{X}$ and $\mathrm{Y}$, it either attacks its prey with probabilities $p$ and $q$, or ignores its prey with probabilities 1- $p$ and 1- $q$, respectively. For simplic-

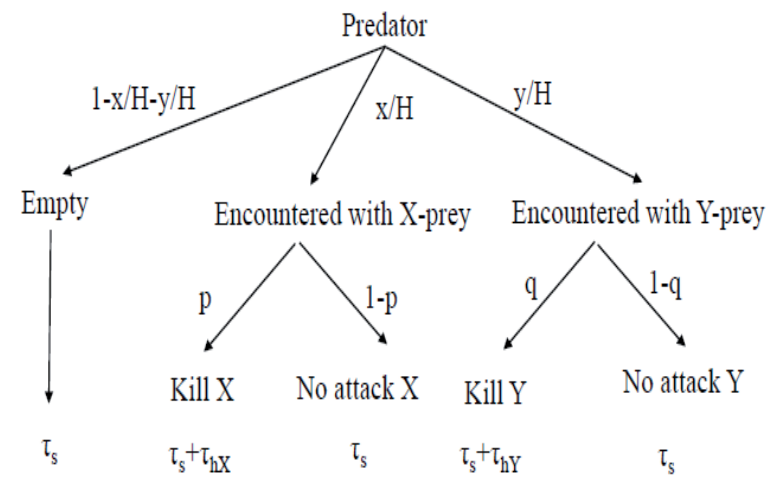

Figure 4. Predator decision tree for two types of prey. The first level describes the encounter events, the second level gives the predator's prey preference. 
ity, we assume the predator is always successful and there are no recognition and missed attack times. The handling times of prey $\mathrm{X}$ and $\mathrm{Y}$ are denoted by $\tau_{h X}$ and $\tau_{h Y}$, respectively. This situation is described in Figure 4.

Based on Figure 4, now the functional responses for prey $\mathrm{X}$ and $\mathrm{Y}$ are

$$
F_{X}=\frac{p x}{\tau_{s} H+\tau_{h X} p x+\tau_{h Y} q y} \text { and } F_{Y}=\frac{q y}{\tau_{s} H+\tau_{h X} p x+\tau_{h X} q y},
$$

respectively.

Application 7. Prey refuge. ${ }^{1}$ Consider one prey and one predator species, and assume that there is no interaction between predators, and the encounter probability depends on prey density denoted by $x$ again. (As in Application 1, let $x / H$ be the encounter probability, where $H$ is the number of perception ranges.) Now, the prey individuals use an absolutely safe refuge, namely, each prey individual spends a proportion $s$ of its time in the open and $1-s$ in the refuge. When the predator has arrived, the prey is in its refuge, then the predator starts a new search, since the refuge is absolutely safe. When the predator has arrived and the prey is in the open area, then the predator always attacks its prey, and kills it with probability $k$. All these activities need time $\tau_{k}=\tau_{s}+\tau_{h}$, where $\tau_{s}$ denotes the searching time, and $\tau_{h}$ is the handling. Here, for simplicity, we assume that the attack does not take time (see Fig. 5). Now the functional response is

$$
F=\frac{k x}{\tau_{s} H+\tau_{h} s k x} .
$$

In the next application, I call the attention to the fact that the decision tree method is appropriate to take into account the behavior of the prey (not only predator's prey preference), so this method is apt to provide a behavior dependent functional response. However, when prey and predator behavior together determine the functional response, then the term "game tree" is more suitable than the decision tree.

Application 8. Dispersal-foraging game. This game is a combination of prey habitat selection between two patch types and optimal foraging approaches (Garay et al. 2015b). Prey's patch preference and predator's behavior determine the prey's survival rate. The predator's energy gain depends on local prey density in both types of exhaustible patches and on leaving time of the predator. In this situation, the decision tree can be considered as a game tree, since at fixed strategies of predator and prey population, the decision tree gives the payoff functions (survival rate for prey, and functional response for predator, see Fig. 6). In the one prey-one predator system, for the simplicity we assume that there are two types of well mixed habitats with different local prey densities, $x_{1}(s)$ and $x_{2}(s)$, where $s$ describes the strategy of habitat use of prey. These habitats are denoted by A-H and B-H, respectively. Denote by $d_{1}(s)$ and $d_{2}(s)$ the relative frequencies of these types of habitat. We can assume that the random predator

${ }^{1}$ I note that this example is based on Cressman and Garay (2009).

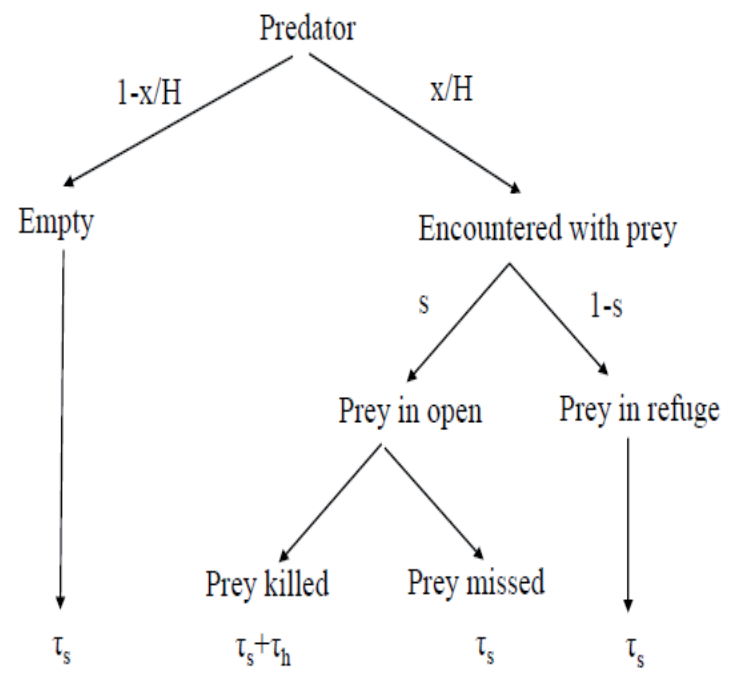

Figure 5. Now the antipredator behavior is refuge usage. When the predator has arrived in a perception range, and there is a prey, the latter is in refuge or in open with probability $s$, and $1-s$, respectively.

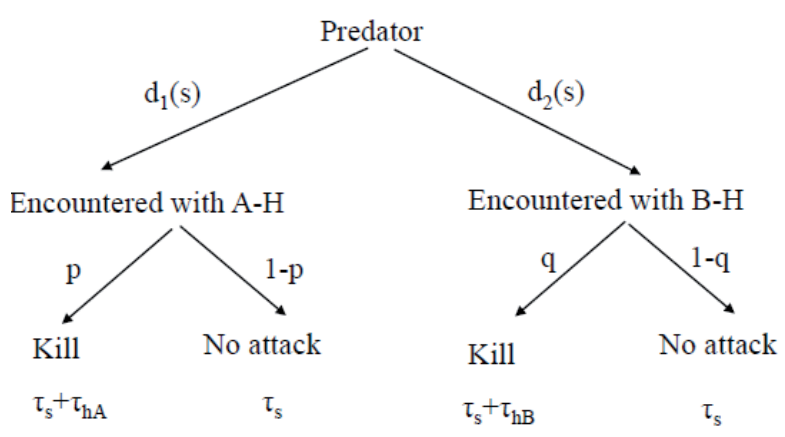

Figure 6. For simplicity, now we assume that all perception ranges are occupied by prey, i.e., there is no empty perception range. Now the antipredator behavior of prey is habitat use. Before the predator has arrived, prey have occupied the different habitats, so the encounter probabilities are determined by the prey.

can find these habitats with respective probabilities $d_{1}(s)$ and $d_{2}(s)$, since the habitats are well-mixed. If predator has found A-H and B-H, it either attacks its prey with probabilities $p$ and $q$, or ignores its prey with probabilities $1-p$ and $1-q$, respectively. The handling times of prey in A-H and $\mathrm{B}-\mathrm{H}$ are denoted by $\tau_{h A}$ and $\tau_{h B}$, respectively.

Now the prey and predator dependent functional response is

$$
F(s, p, q)=\frac{d_{1}(s) p+d_{2}(s) q}{\tau_{s} H+\tau_{h A} d_{1}(s) p x+\tau_{h B} d_{2}(s) q x} x .
$$




\section{Deterministic derivation methods}

In this section we overview the deterministic methods used for the derivation of functional responses.

\subsection{Deterministic method for the stage of predator with fixed prey density and time constraint}

Consider, now an infinitely large predator population, where each predator has $k$ different stages. The transition rate between stages is given by the assumption of infinitely large population, the rule of mass action (Van der Meer and Smallegange 2009) as in chemical systems (Huisman and de Boer 1997, Real 1977). Thus, the encounter rate between predators and prey is proportional to the product of the number of predator in searching stage and the density of prey. The transition rate from handling stage to searching stage is constant and inversely proportional to the handling time. From mathematical point of view, the latter idea relies on the assumption that the distribution of handling time is exponential, thus the average time duration of each handling period of each predator individual is $1 / \tau_{h}$.

To demonstrate this deterministic derivation method, let us follow the original assumption of Holling (see Section 2.1). Let us consider only two stages of predator, searching $(S)$ and handling $(H)$, which exclude each other and both need time, $\tau_{s}$ and $\tau_{h}$, respectively. The number of predators $(Y)$ does not change during time period of predation $T$, consequently

$$
S=Y-H,
$$

where $S$ and $H$ are the numbers of predators at stages searching and handling, respectively. Furthermore, during $T$ the density of prey $(x)$ and finding rate of a prey $(a)$ do not change and predator always successfully kills its attacked prey, thus $a x$ is the killing rate during $T$.

Now we can give a differential equation model on the stages of all predators:

$$
\begin{aligned}
& \frac{d S}{d t}=-\frac{a x}{\tau_{S}} S+\frac{1}{\tau_{h}} H . \\
& \frac{d H}{d t}=\frac{a x}{\tau_{S}} S-\frac{1}{\tau_{h}} H .
\end{aligned}
$$

Indeed, as Figure 7 shows, the rate between searching and handling is $a x / \tau_{s}$, since a predator has to finish its actual search (with probability $1 / \tau_{s}$ ) and find its prey with probability $a x$ and kill its found prey with probability 1 . Furthermore, at each time unit, the $1 / \tau_{h}$ part of the handling predators finish the handling stage and start a new search.

In the long run, there exists a mixed stable equilibrium, since if all predators are in the searching stage then a fraction of them will start handling stage, and contrarily, if all predators are in handling stage then a fraction of them will start searching stage. The equilibrium is given by

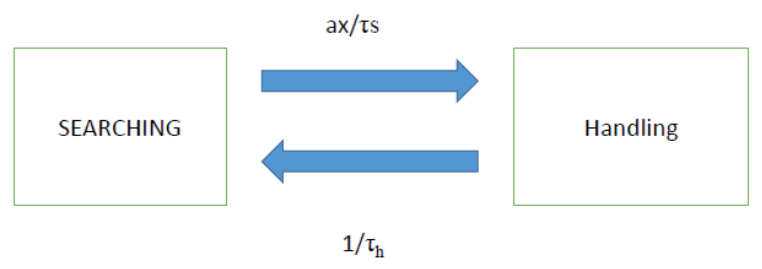

Figure 7. Two-stage model: when in average the searching and handling stages take times $\tau_{s}$ and $\tau_{h}$, respectively, and both times are exponentially distributed.

$\frac{a x}{\tau_{S}}(Y-H)=\frac{1}{\tau_{h}} H$,

which easily yields

$\frac{a x \tau_{h}}{\tau_{S}+a x \tau_{h}} Y=H$

Finally, since the functional response considers a single predator, in this way we also obtain the Holling type II functional response. For more mathematical details of population-level differential equation models, see e.g., Metz and Diekmann (1986).

This way of derivation of functional response was applied to a range of situations. For instance, Ruxton et al. (1992) and Van der Meer and Smallegange (2009) by using this derivation method obtained a Beddington-DeAngelis-like functional response for the case when predators fight with each other. Kooijman (2006), when a prey type is not edible, also derived a Beddington-DeAngelis-like functional response. Later, Geritz and Gyllenberg (2012) also found a Beddington - DeAngelis functional response involving prey refuges instead of the usual interference between predators. For the plant-bees interaction, Fishman and Hadany (2010) found an analytical approximation of the Beddington -DeAngelis functional response. Furthermore, van Leeuwen et al. (2007), taking into account densities of two prey types, obtained Holling types II and III (Real 1977) functional responses

In ecological game theory, the above stage dynamic method is often used when the interaction takes time. For instance, this method is applied in a predator-prey system where predators fight for captured prey and fighting for prey is modelled by a hawk-dove game dynamics (Auger et al. 2002, Kooi 2015). The adaptive dynamics approach as an evolutionary research line also uses this functional response derivation method in model building (Dercole 2016).

I note that this deterministic mass action method is widely used in evolutionary game theory under time constraint (Broom and Rychtar 2013, Krivan and Cressman 2017) as well.

\subsection{Deterministic method for decreasing prey density without time constraint}

In this subsection, we recall some classical results reviewed by Royama (1971), see also Hassell (1978, Appendix 
I). We will see how the exponential function arises in the study of functional response (e.g., Ivlev 1961, Nicholson and Bailey 1935, Royama 1971). Only in this subsection we assume that during the predation period $T$ the density $x$ of prey is decreased by predators, but the predator density is constant, $Y$. Let us suppose that at prey density $x, f(x)$ is the number of prey killed by a predator in unit time, considered as an "instantaneous functional response" in the sense that the implied time-dependent prey density is described in terms of the differential equation

$\frac{d x}{d t}=-f(x) Y$.

By solving this differential equation with initial condition $x(0)=x_{0}$, we can calculate an "average functional response" over the time duration $T$, defined as

$$
\frac{x_{0}-x(T)}{T} .
$$

For illustration, let us consider the particular case when $f(x)=a x$, with some constant $a$. Then, from (11) by integration we obtain

$$
\frac{1}{a} \int_{x_{0}}^{x} \frac{d u}{u}=-Y \int_{0}^{T} d s
$$

providing the average functional response

$$
F(x)=\frac{x_{0}-x(T)}{T}=\frac{x_{0}}{T}[1-\exp (-a Y T)]
$$

Observe that this corresponds to the Nicholson-Bailey (1935) "competition equation" (see Royama 1971, p. 11-13). Finally we note, if the instantaneous functional response is not a linear function of prey density, then we get a different average functional response. For instance, let us start from Ivlev (1961) function

$$
f(x)=b[1-\exp (-a x)]
$$

where $a$ and $b$ are positive constants. Then, applying the above differential equation method we get the following average functional response

$$
F(x)=-\frac{1}{a T} \ln \left\{\left[1-\exp \left(-a x_{0}\right)\right] \exp (-a b Y T)+\exp \left(-a x_{0}\right)\right\} .
$$

Using this derivation method, Okuyama and Ruyle (2011) derived Holling types II, III, Beddigton-DeAngelis and ratio dependent functional responses. Moreover, Aljetlawi et al. (2004) derived a functional response function that accounted for both predator and prey size. Furthermore, Cabello et al. (2007) introduced new expressions for Holling type III in terms of a new parameter called entomophagous potential (of a parasitoid or predator), which is important from the view point of biological pest control (e.g., Fernandez-Maldonado et al. 2017).

\section{A short note on the connection between deterministic and stochastic approaches}

As we already have shown, different derivation methods may give the same functional response. For instance, Van der Meer and Smallegange (2009), using continuous time Markov model for a finite population and deterministic mass action method for infinite populations, derived the same functional response. The connection between finite Markov processes and differential equations is given by Kolmogorov (1931), to characterize the stochastic process. In a series of papers, Metz and Van Batenburg (1985), Metz and Diekmann (1986) and Metz et al. (1988) formulated the full continuous versions of the finite stochastic models, and studied their asymptotic behavior using differential equations. Sabelis (1990), applying the same method, built a model on the assumption that gut fullness is the sole internal state variable determining the attack rate. Dawes and Souza (2013) using the same method provided functional responses: the Holling type I (when handling time is very short), type II (when handling time is long compared to searching time) and type III (when the mortality of the prey first increases with prey density at low densities, and then decreases at higher prey densities, so that the response curve has a characteristic $S$-shaped form)

I note that in physics, starting from a microscopic stochastic model (individual level model), taking a limit in the densities of prey and predator, McKane and Newman (2005) arrived at a mean field (differential equation) model to give the functional response.

\section{Discussion}

First of all, Holling type II is the experimentally most widely used functional response (Skalski and Gilliam 2001) and also very much used in theoretical investigations. I emphasize that all derivation methods are able to derive the Holling type II functional response. Consequently, all reviewed derivation methods have proved their usefulness in theoretical ecology.

Now I am in the position to make clear what until now has been the unannounced aim of this review. Predation is a pure counter interested phenomenon, since predator has to kill its prey for living and this prey has to survive (or avoid) the predator attacks, thus each of them survives only at a cost to the other. The predator success depends on the behavior of both opponents, so in general, from mathematical point of view, the predation process can be described in terms of game theory, which is the mathematical tool to understand the conflicts of counter-interested species (Lima 2002). To define a game, we need to give the strategy set (now the behavior of opponents), and the payoff functions. For the predator the payoff function is the numerical response. For the prey the 
payoff function is the probability of survival. Both payoff functions can be defined in terms of the functional response, which describes the predator success. Consequently, during the derivation of ecological games, the derivation methods of behavior dependent functional responses are an essential component of the definition of the payoff functions of prey and predator as well. Thus, the unannounced aim of this review is to assist the development of the research line of ecological games. To make clear why, in general, evolutionary game theory is unavoidable in foraging theory, let us consider the problem of foraging under predation risk (Lima and Dill 1990, Scharf et al. 2011). For reproduction, animals must collected food and survive, thus if the forager is not a top predator, then the food collected per unit time is not a sufficient factor to measure fitness. The antipredator behavior (e.g., vigilance) also needs time, and the time constraint (if antipredator behavior and feeding exclude each other) implies a trade-off between food and safety. I note that evolutionary game theory has already been applied to the problem of vigilance (e.g., Brown 1999, Sirot 2012).

Finally, time constraint is an essential motive in the functional response. Recently, much attention has been focused on the role of time constraints in evolutionary game theory which are not directly connected with functional response. Now the question arises: which derivation methods have been already used in evolutionary games? I mention only two research lines. The first one considers the problem of kleptoparasitism. Broom and his coauthors have several papers on this problem and they use two derivation methods. They most often use the stage differential equations to find the steady state of the predator population, see Section 4.1 (e.g., Hadjichrysanthou and Broom 2012, Spencer and Broom 2018). They also use Markov processes to describe the stationary state of the predator population (Broom et al. 2010, Yates and Broom 2007). The second research line concerns matrix games under time constraint, where the stage differential equations are also used to find the steady state of the players (Cressman and Krivan 2019, Krivan and Cressman 2017), moreover, the Markov processes are also applied to describe the stationary state of the players (Garay et al. 2016). Based on all this, I hope the derivation methods overviewed here will be useful not only in ecology, but also in evolutionary game theory when the time constraints take place.

Acknowledgements. This work was funded by National Research, Development and Innovation Office Grants (NKFI-K119347) and GINOP-2.3.2-15-2016-00057 ("In the light of evolution"). The author is also grateful for the valuable comments of the Editor and the anonymous reviewers.

\section{References}

Abrams, P.A. 2014. Why ratio dependence is (still) a bad model of predation. Biol. Rev. 90:794-814.

Abrams, P.A. and L.R. Ginzburg. 2000. The nature of predation: prey dependent, ratio dependent or neither? TREE 15:337-341.
Abrams, P.A., H. Matsuda and Y. Harada. 1993. Evolutionarily unstable fitness maxima and stable fitness minima of continuous traits. Evol. Ecol. 7:465-487.

Aljetlawi, A.A., E. Sparrevik and K. Leonardsson. 2004. Preypredator size-dependent functional response: derivation and rescaling to the real world. J. Anim. Ecol. 73:239-252.

Andersson, M. 1981. On optimal predator search. Theor. Pop. Biol. 19:58-86.

Andrews, J.F. 1968. A mathematical model for the continuous culture of microorganisms utilizing inhibitory substrates. Biotechnol. Bioeng. 10:707-723.

Arditi, R. and L.R. Ginzburg. 1989. Coupling in predator-prey dynamics: ratio-dependence. J. Theor. Biol. 139:311-326.

Arditi, R. and L.R. Ginzburg. 2012. How Species Interact: Altering the Standard View on Trophic Ecology. Oxford Univ. Press, New York

Arditi, R., L.R. Ginzburg and H.R. Akcakaya. 1991. Functional responses and heterogeneities: an experimental test with cladocerans. Oikos 60:69-75.

Auger, P., R.B. de la Parra, S. Moran and E. Sánchez. 2002. A predator-prey model with predators using hawk and dove tactics. Math. Biosci. 177-178:185-200.

Auger, P., R.B. de la Parra, S. Morand and E. Sánchez. 2002. A predator-prey model with predators using hawk and dove tactics. Math. Biosci. 177:185-200.

Baker, D.J., R.A. Stillman, B.M Smith. J.M. Bullock and K.J. Norris. 2010. Vigilance and the functional response of granivorous foragers. Funct. Ecol. 24:1281-1290.

Barta, Z. and L.A. Giraldeau. 2000. Daily patterns of optimal producer and scrounger use under predation hazard: A state-dependent dynamic game analysis. Am. Nat. 155:570-582.

Baudrot, V., A. Perasso, C. Fritsch, P. Giraudoux and F. Raoul. 2016 The adaptation of generalist predators' diet in a multi-prey context: insights from new functional responses. Ecology 97:1832-1841.

Beddington, J.R. 1975. Mutual interference between parasites or predators and its effect on searching efficiency. J. Anim. Ecol. 44:331-340.

Billiard, S., V. Bansaye and J.R. Chazottes. 2018. Rejuvenating functional responses with renewal theory. J. Roy. Soc. Interface $15: 146$.

Boon, B. and H. Landelout. 1962. Kinetics of nitrite oxidation by nitrobacter winogradskyi. Biochem. J. 85:440-447.

Broom, M., M.L. Crowe, M.R. Fitzgerald and J. Rychtář. 2010. The stochastic modelling of kleptoparasitism using a Markov process. J. Theor. Biol. 264:266-272.

Broom M. and V. Křivan. 2018. Biology and evolutionary games. In: T. Basar and G. Zaccour (eds), Handbook of Dynamic Game Theory. Springer, Cham. pp. 1039-1077.

Broom, M. and J. Rychtar. 2013. Game-theoretical Models in Biology. CRC, Boca Raton, FL.

Brown, J.S. 1999. Vigilance, patch use and habitat selection: Foraging under predation risk. Evol. Ecol. Res. 1:49-71.

Brown, J.S. J.W. Laundré and M. Gurung. 1999. The ecology of fear: Optimal foraging, game theory, and trophic interactions. $J$. Mammal. 80:385-399.

Brown, J.S. and T.L. Vincent. 1992. Organization of predator-prey communities as an evolutionary game. Evolution 46:1269-1283.

Cabello, T., M. Gámez and Z. Varga, 2007. An improvement of the Holling type III functional response in entomophagous model. $J$. Biol. Syst. 15:515- 524. 
Casas, J., W.S.C. Gurney, R. Nisbet and O. Roux. 1993. A probabilistic model for the functional response of a parasitoid at the behavioural time-scale. J. Anim. Ecol. 62:194-204.

Charnov, E.L. 1976. Optimal foraging: attack strategy of a mantid. Am. Nat. 110:141-151.

Colombo, M. 2013. Determinants of winter kill rates of wolves in Scandinavia. Second cycle, A2E. Umea: SLU, Dept. of Wildlife, Fish and Environmental Studies

Cosner, C., D.L. DeAngelis, J.S. Ault and D.B. Olson. 1999. Effects of spatial grouping on the functional response of predators. Theor. Pop. Biol. 56:65-75.

Cressman, R. and J. Garay. 2009. A predator-prey refuge system: Evolutionary stability in ecological systems. Theor. Pop. Biol. $76: 248-257$.

Cressman, R. and J. Garay. 2011. The effects of opportunistic and intentional predators on the herding behavior of prey. Ecology 92:432-440.

Cressman, R. and V. Krivan. 2019. Bimatrix games that include interaction times alter the evolutionary outcome: The owner-intruder game. J. Theor. Biol. 460:262-273.

Cressman, R., V. Krivan and J. Garay. 2004. Ideal free distributions, evolutionary games, and population dynamics in multiple-species environments. Am. Nat. 164:473-489.

Cressman R, V. Krivan, J.S. Brown and J. Garay. 2014. Gametheoretic methods for functional response and optimal foraging behavior. PLoS ONE 9(2), e88773

Dawkins, M. 1971. Shifts of 'attention' in chicks during feeding. Anim. Behav. 19:575-582.

Dawes, J.H.P. and M.O. Souza. 2013. A derivation of Holling's type I, II and III functional responses in predator-prey systems. $J$. Theor. Biol. 327:11-22.

DeAngelis, D.L., R. A. Goldstein and R.V.O’Neill. 1975. A Model for tropic interaction. Ecology 56:881-892.

Dercole, F. 2016. The ecology of asexual pairwise interactions: the generalized law of mass action. Theor. Ecol. 9:299-321.

Edwards, V.H. 1970. Influence of high substrate concentrations on microbial kinetics, Biotechnol. Bioeng. 12:679-712.

Erichsen, J.T., J.R. Krebs and A.I. Houston. 1980. Optimal foraging and cryptic prey. J. Anim. Ecol. 49:271-276.

Eshel, I. 1978. On a prey-predator nonzero-sum game and the evolution of gregarious behavior of evasive prey. Am. Nat. 112:787-795

Fenlon, J.S. and M.J. Faddy. 2006. Modelling predation in functional response. Ecol. Model. 198:154-162.

Fernandez-Maldonado, F.J., J.R. Gallego, M. Gamez, Z. Varga, J. Garay and T. Cabello 2017. Cannibalism: Do risks of fighting and reprisal reduce predatory rates? Community Ecol. 18:87-96.

Fishman, M.A. and L. Hadany. 2010. Plant-pollinator population dynamics. Theor. Pop. Biol. 78:270-277.

Fretwell, D.S. and H.L. Lucas. 1970. On territorial behavior and other factors influencing habitat distribution in birds. Acta Biotheoretica 19:16-32.

Fryxell, J.M., A. Mosser, A.R.E. Sinclair and C. Packer. 2007. Group formation stabilizes predator-prey dynamics. Nature 449:10411043

Gagniuc, P.A. 2017. Markov Chains: From Theory to Implementation and Experimentation. Wiley, Hoboken.

Garay, J., R. Cressman, F. Xu, Z. Varga and T. Cabello. 2015b. Optimal forager against Ideal Free Distributed prey. Am. Nat. 186:111-122
Garay, J., R. Cressman and Z. Varga, 2003. Optimal behaviour of honey bees based on imitation at fixed densities. Community Ecol. 4:219-224.

Garay J., V. Császár and T.F. Móri 2016. Evolutionary stability for matrix games under time constraints. J. Theor. Biol. 415:1-12.

Garay, J. and F.T. Móri, 2010. When is the opportunism remunerative? Community Ecol. 11:160-170.

Garay, J., Z. Varga, T. Cabello and M. Gamez. 2012. Optimal nutrient foraging strategy of an omnivore: Liebig's law determining numerical response. J. Theor. Biol. 310:31-42.

Garay J., Z. Varga, M. Gámez and T. Cabello. 2015a. Functional response and population dynamics for fighting predator, based on activity distribution. J. Theor. Biol. 368:74-82.

Garay, J., Z. Varga, T.F., Móri, I. López, M. Gámez, J.R. Gallego and T. Cabello. 2018. Opportunistic random searcher versus intentional search image user. Scientific Reports 8:3336

Garcia-Martín, M., M. Gámez, A. Torres-Ruíz and T. Cabello. 2008. Functional response of Chelonus oculator (Hy: Braconidae) to temperature, and its consequences to parasitims. Community Ecol. 9:45-51.

Geritz, S. and M. Gyllenberg. 2012. A mechanistic derivation of the DeAngelis-Beddington functional response. J. Theor. Biol. 314:106-108

Getty, T. 1985. Discriminability and the sigmoid functional response: How optimal foragers could stabilize model-mimic complexes. Am. Nat. 125:239-256.

Hadjichrysanthou, C. and M. Broom. 2012. When should animals share food? Game theory applied to kleptoparasitic populations with food sharing. Behav. Ecol. 23:977-991.

Hammill, E., O.L. Petchey and B.R. Anholt. 2010. Predator functional response changed by induced defenses in prey. Am. Nat. 176:723-731

Hammond, J.I., B. Luttbeg and A. Sih. 2007. Predator and prey space use: Dragonflies and tadpoles in an interactive game. Ecology $88: 1525-1535$.

Hassell, M.P. 1978. The Dynamics of Arthropod Predator-prey Systems. Princeton Univ. Press, New Jersey.

Hayward, M.W. and G.I. Kerley. 2005. Prey preference of the lions (Panthera leo). J. Zool. London 267:309-322.

Holland, H.J.N. and D.L. DeAngelis. 2010. A consumer-resource approach to the density dependent population dynamics of mutualism. Ecology 91:1286-1295.

Holland, J.N., D.L. DeAngelis and J.L. Bronstein. 2002. Population dynamics and mutualism: functional responses of benefits and costs. Am. Nat. 159:231-244.

Holling, C.S. 1959a. The components of predation as revealed by a study of small-mammal predation of the European pine sawfly. Can. Entom. 91:293-320.

Holling, C.S. 1959b. Some characteristics of simple types of predation and parasitism. Can. Entom. 91:385-98.

Holling, C.S. 1966. The functional response of invertebrate predators to prey density. Mem. Entom. Soc. Can. 48:5-88.

Holt, R.D. 1985. Population dynamics in two-patch environments: Some anomalous consequences of an optimal habitat distribution. Theor. Pop. Biol. 28:181-208.

Holt, R.D. and M.B. Bonsall. 2017. Apparent competition. Ann. Rev. Ecol. Evol. Syst. 48:447-471.

Hossie, T.J. and D.L. Murray. 2010. You can't run but you can hide: refuge use in frog tadpoles elicits density-dependent predation by dragonfly larvae. Oecologia 163:395-404. 
Hossie, T.J. and D.L. Murray. 2016. Spatial arrangement of prey affects the shape of ratio-dependent functional response in strongly antagonistic predators. Ecology 97:834-841.

Houston, A.I. and J.M. McNamara. 1985. A general theory of central place foraging for single-prey loaders. Theor. Pop. Biol. 28:233-262.

Houston, A.I. and J.M. McNamara. 1999. Models of Adaptive Behaviour. Cambridge

Hugie, D.M. and L.M. Dill, 1994. Fish and game: a game theoretic approach to habitat selection by predators and prey. J. Fish Biol. 45:151-169.

Huisman, G. and R.J. de Boer, 1997. A formal derivation of the “Beddington”'functional response. J. Theor. Biol. 185:389-400.

Inger, R., S. Bearhop, J.A. Robinson and G. Ruxton. 2006. Prey choice affects the trade-off balance between predation and starvation in an avian herbivore. Anim. Behav. 71:1335-1341.

Ivlev, V.S. 1961. Experimental Ecology of the Feeding of Fishes. Yale Univ. Press, New Haven, CT.

Iyengar, E.V. 2008. Kleptoparasitic interactions throughout the animal kingdom and a re-evaluation, based on participant mobility, of the conditions promoting the evolution of kleptoparasitism. Biol. J. Linnean Soc. 93:745-762,

Jeschke, J.M., M. Kopp and R. Tollrian. 2002. Predator functional responses: Discriminating between handling and digesting prey. Ecol. Monogr. 72:95-112.

Johns, M.V. and R.G. Miller. 1963. Average renewal loss rates. Ann. Math. Stat. 34:396-401.

Jost, C. and S.P. Ellner. 2000. Testing for predator dependence in predator-prey dynamics: a non-parametric approach. Proc. $R$. Soc. B. 267:1611-1620.

Jost, C., G. Devulder, J.A. Vucetich, R.O. Peterson and R. Arditi. 2005. The wolves of Island Royale display scale-invariant satiation and ratio-dependent predation on moose. J. Anim. Ecol. 74:809-816.

Kalinoski, R.M. and J.P. DeLong, 2016. Beyond body mass: how prey traits improve predictions of functional response parameters. Oecologia 180:543-550.

Kannan, D. 1983. A Markov chain analysis of predator strategy in a model-mimic system. Bull. Math. Biol. 45:347-400.

Kolmogorov, A. 1931 Über die analytischen Methoden in der Wahrscheinlichkeitsrechnung" (On Analytical Methods in the Theory of Probability). Mathematische Annalen 104:415-458.

Kohlmann, S.G., J.H. Matis and K.L. Risenhoover. 1999. Estimating handling times for herbivore diets: a statistical method using the gamma distribution. J. Anim. Ecol. 68:638-645.

Kondoh, M. 2003. Foraging adaptation and the relationship between food-web complexity and stability. Science 299:1388-1391.

Kondoh, M. 2006. Does foraging adaptation create the positive complexity-stability relationship in realistic food-web structure? $J$. Theor. Biol. 238:646-651.

Kondoh, M. 2007. Anti-predator defence and the complexity-stability relationship of food webs. Proc. R. Soc. B. 274:1617-1624.

Kooi, B.W. 2015. Modelling the dynamics of traits involved in fighting-predators-prey system. J. Math. Biol. 71:1575-1605.

Kooijman, S.A.L.M. 2006. Pseudo-faeces production in bivalves. $J$ Sea Res. 56:103-106.

Kotler, B. and W.A. Mitchell. 1995. The effect of costly information in diet choice. Evol. Ecol. 9:18-29.

Kratina, P., M. Vos, A. Bateman and B.R. Anholt. 2009. Functiona responses modified by predator density. Oecologia 159:425-433.
Krivan, V. and R. Cressman. 2009. On evolutionary stability in preypredator models with fast behavioral dynamics. Evol. Ecol. Res. $11: 227-251$

Krivan, V. and R. Cressman, 2017. Interaction times change evolutionary outcomes: Two-player matrix games. J. Theor. Biol. 416:199-207.

Krivan, V., R. Cressman and C. Schneider, 2008. The ideal free distribution: A review and synthesis of the game-theoretic perspective. Theor. Pop. Biol. 73:403-425.

Krivan, V. and O.J. Schmitz, 2003. Adaptive foraging and flexible food web topology. Evol. Ecol. Res. 5:623-652.

Krivan, V. and A. Sikder. 1999. Optimal foraging and predator-prey dynamics II. Theor. Popul. Biol., 55:111-126.

Lester, P.J. and R. Harmsen. 2002. Functional and numerical responses do not always indicate the most effective predator for biological control: an analysis of two predators in a two-prey system. $J$. Appl. Ecol. 39:455-468.

Lett, C., P. Auger and J.M. Gaillard. 2004. Continuous cycling of grouped vs. solitary strategy frequencies in a predator-prey model. Theor. Pop. Biol. 65:263-270.

Lima, S.L. 2002. Putting predators back into behavioral predatorprey interactions. TREE 17:70-75.

Lima, S.L. and L.M. Dill. 1990. Behavioral decisions made under the risk of predation: a review and prospectus. Can. J. Zool. 68:619-640

McCoy, M.W., B.M. Bolker, K.M. Warkentin and J.R. Vonesh. 2011 Predicting predation through prey ontogeny using size-dependent functional response models. Am. Nat. 177:752-766.

MacDougall, A. and M. Dawkins. 1998. Müllerian mimicry and the receiver psychology of imperfect discrimination. Anim. Behav. $55: 1281-1288$

McKane, A.J. and T.J. Newman 2005. Predator-prey cycles from resonant amplification of demographic stochasticity. Phys. Rev. Lett. 94:218102.

McNair, J.N. 1987. Stability effects of prey refuges with entry-exit dynamics. J. Theor. Biol. 125:449-464.

McNamara, J.M. 1979. A generalized model of optimal diets. Theor Pop. Biol. 15:159-170.

McNamara, J.M. 1985. An optimal sequential policy for controlling a Markov renewal process. J. Appl. Prob. 22:324-335.

McNamara, J.M., A.I. Houston and E.J. Collins. 2006. Optimality models in behavioral biology SIAM Rev. 43:413-466.

Merilaita, S. 2006. Frequency-dependent predation and maintenance of prey polymorphism. J. Evol. Biol. 19:2022-2030.

Metz, J.A.J. and O. Diekmann. 1986. The Dynamics of Physiologically Structured Populations. Lecture Notes in Biomathematics Volume 68. Springer, Berlin

Metz, J.A.J. and F.H.D. van Batenburg. 1985. Holling's "hungry mantid" model for the invertibrate functional response considered as a Markov process. Part I: The full model and some of its limits. J. Math. Biol. 22: 209-238.

Metz J.A.J, M.W. Sabelis and J.H. Kuchlein. 1988. Sources of variation in predation rates at high prey densities: an analytic model and a mite example. Exp. Appl. Acarol. 5:187-205.

Molla, H., R. Md. Sabiar and S. Sarwardi. 2018. Dynamics of a predator-prey model with Holling type II functional response incorporating a prey refuge depending on both the species. Int. J. Nonlin. Sci. Num. Simul. 20:1-16,

Murdoch, W.W., S. Avery and M.E.B. Smyth. 1975. Switching in predatory fish. Ecology 56:1094-1105. 
Nicholson, A.J. and V.A. Bailey. 1935. The Balance of Animal Populations.-Part I. Proc. Zool. Soc. London 3:551-598.

Oaten, A. 1977. Optimal foraging in patches: a case for stochasticity. Theor. Pop. Biol. 12:263-285.

Oaten, A. and W.W. Murdoch 1975. Switching, functional response, and stability in predator-prey systems. Am. Nat. 109:299-318.

Okuyama, T. and R.L. Ruyle. 2011. Solutions for functional response experiments. Acta Oecol. 37:512-516.

Pavlic, T.P. and K.M. Passino. 2011. Generalizing foraging theory for analysis and design. Int. J. Robot. Res. 30:505-523.

Pawar, S., A.I. Dell and V.M. Savage. 2012. Dimensionality of consumer search space drives trophic interaction strengths. Nature 486:485-489.

Punzalan, D., F.H. Rodd and K.A. Hughes. 2005. Perceptual processes and the maintenance of polymorphism through frequency dependent predation. Evol. Ecol. 19:303-32.

Real, L.A. 1977. The kinetics of functional response. Am. Nat. 111: 289-300.

Rogers, D. 1972. Random search and insect population models. $J$. Anim. Ecol. 41:369-383.

Royama, T. 1971. A comparative study of models for predation and parasitism Res. Pop. Ecol. 13:1-91.

Ruxton, G.D. and W.S.C. Gurney, 1994. Deriving the functional response without assuming homogeneity. Am. Nat. 144:537-541.

Ruxton, G.D., W.S.C. Gurney and A.M. De Roos. 1992. Interference and generation cycles. Theor. Pop. Biol. 42:235-253,

Sabelis, M.W. 1990. How to analyse prey preference when prey density varies? A new method to discriminate between effects of gut fullness and prey type composition. Oecologia 82:289-298.

Saleem, M., A.H. Sadiyal, P. Edi and P.R. Arora. 2006. Evolutionarily stable strategy for defensive switching. Appl. Math. Comp. 177:697-713.

Scharf, I., Y. Lubin and O. Ovadia. 2011. Foraging decisions and behavioural flexibility in trap-building predators: a review. Biol. Rev. 86:626-639.

Sherratt T. N. 2002. The evolution of imperfect mimicry. Behav. Ecol. 13:821-826.

Sih, A. 1987. Prey refuges and predator-prey stability. Theor. Pop. Biol. 31:1-12.

Sirot, E. 2012. Negotiation may lead selfish individuals to cooperate: the example of the collective vigilance game. Proc. $R$. Soc. B. 279:2862-2867.

Skalski, G.T. and J.F. Gilliam. 2001. Functional responses with predator interference: viable alternatives to the Holling type II model. Ecology 82:3083-3092.

Smart, S.L., R.A. Stillman and K.J. Norris. 2008. Measuring the functional responses of farmland birds: an example for a declining seed-feeding bunting. J. Anim. Ecol. 77:687-695.

Solomon, M.E. 1949. The natural control of animal populations. $J$. Anim. Ecol. 18:1-35.

Spalinger, D.E. and N.T. Hobbs. 1992. Mechanisms of foraging in mammalian herbivores: New models of functional response. Am. Nat. 140:325-348.

Spencer, R. and M. Broom. 2018. A game-theoretical model of kleptoparasitic behavior in an urban gull (Laridae) population. Behav. Ecol. 29:60-78.

Stephens, D.W. and J.R. Krebs. 1986. Foraging Theory. Princeton Univ. Press, Princeton.

Tellez, M.M., G. Tapia, M. Gámez, T. Cabello and H.F. Van Emden. 2009. Predation of Bradysia sp. (Dip.: Sciaridae), Liriomyza trifolii (Burgess in Comstock 1880) (Dip.: Agromyzidae) and Bemisia tabaci (Gennadius 1889) (Hom.: Aleyrodidae) by Coenosia attenuata Stein 1903 (Dip.: Muscidae) in greenhouse crops. Europ. J. Entom. 106:199-204.

Tinbergen, L. 1960. The natural control of insects in pinewoods. 1. Factors influencing the intensity of predation by songbirds. Archives Néerlandaises de Zoologie 13:265-343.

Treves, A. 2000. Theory and method in studies of vigilance and aggregation. Anim. Behav. 60:711-722.

Toscano, B.J., B. Newsome and B.D. Griffen. 2014. Parasite modification of predator functional response. Oecologia 175:345-352.

Turelli, M., J.H. Gillespie and T.W. Schoener. 1982. The fallacy of the fallacy of the averages in ecological optimization theory. Am. Nat. 119:879-884.

Uchida, S., B. Drossel and U. Brose. 2007. The structure of food webs with adaptive behavior. Ecol. Model. 206:263-276.

van Baalen, M., V.Krivan, P.C.J. van Rijn and M.W. Sabelis. 2001. Alternative food, switching predators, and the persistence of predator-prey systems. Am. Nat. 157:512-524.

van Baalen, M. and M.W. Sabelis. 1993. Coevolution of patch selection strategies of predator and prey and the consequences for ecological stability. Am. Nat. 142:646-670.

Valdovinos, F.S., R. Ramos-Jiliberto, L. Garay-Narváez, P. Urbani and J.A. Dunne, 2010. Consequences of adaptive behaviour for the structure and dynamics of food webs. Ecol. Lett. 13:1546-1559.

Van der Meer J. and I.M. Smallegange. 2009. A stochastic version of the Beddington-DeAngelis functional response: modelling interference for a finite number of predators. J. Anim. Ecol. 78:134-42.

Van Leeuwen, E., V.A.A. Jansen and P.W. Bright, 2007. How population dynamics shape the functional response in a one-predatortwo-prey system. Ecology 88:1571-1581.

Vardi, R., Z. Abramsky, B.P., Kotler, O. Altstein and M.L. Rosenzweig. 2017. How does the presence of a conspecific individual change the behavioral game that a predator plays with its prey? Oecologia 184:597-607.

Vuchetich, J. A., R.O. Peterson and C.L. Schaffer. 2002. The effect of prey and predator densities on wolf predation. Ecology 83:3003-3013.

Wald, A. 1944. On cumulative sums of random variables. Ann. Math. Stat. 15:283-296.

Wang, Y., H. Wu and S. Sun. 2012. Persistence of pollination mutualisms in plant-pollinator-robber systems. Theor. Pop. Biol. 81:243-250.

Wittenberger, J.F. and J.L. Hunt. 1985. The adaptive significance of coloniality in birds. In: D.S. Farner J.R. King and K.C. Parkes (eds), Avian Biology, vol. 3 Academic Press, New York. pp. 1-75.

Yates, G.E. and M. Broom. 2007. A stochastic model of kleptoparasitism. J. Theor. Biol. 248:480-489.

Yearsley, J.M. 2003. Optimal diet selection, frequency dependence and prey renewal. Theor. Pop. Biol. 64:129-139.

Received January 21, 2019

Revised March 26, 2019

Accepted April 12, 2019

Open Access statement. This is an open-access article distributed under the terms of the Creative Commons Attribution 4.0 International License (https://creativecommons.org/ licenses/by/4.0/), which permits unrestricted use, distribution, and reproduction in any medium, provided the original author and source are credited, a link to the CC License is provided, and changes - if any - are indicated. (SID_1). 